\title{
Adsorption of Rhodamine-B Dye from an Aqueous Solution by Biomass Pine Apple Peel: Kinetics, Equilibrium and Thermodynamic Studies
}

\author{
N Ramulu ${ }^{1}$, V Thirumurugan ${ }^{1 *}$, S Krishnaveni ${ }^{1}$ and R Rajajeyaganthan ${ }^{2}$ \\ ${ }^{1}$ AVVM Sri pushpam college,(Autonomous) poondi, Tamilnadu, India. \\ ${ }^{2} M$ Kumarasamy college of Engineering, Karur, Tamilnadu, India.
}

Received: June 16, 2016; Accepted: June 30, 2016; Published: July 15, 2016

*Corresponding author: V Thirumurugan, AVVM Sri Pushpam college,(Autonomous) poondi, Thanjavur-613501,Tamilnadu, India.

E-mail: drv.thirumurugan@gmail.com

\begin{abstract}
Today there are lots of dyes available commercially. They are used in many industries such as paper, rubber, plastic, cosmetics and especially Textiles. Many methods have been proposed in order to remove color from waste water among which adsorption is more acceptable due to the ability for its use in the large scale. The objectives of this study were to investigate pine apple peel as on inexpensive adsorbent for removal of Rhodamine- $\mathrm{B}$ dye from aqueous solution. In this study the effect of $\mathrm{pH}$, initial concentration, contact time and amount of adsorbent where optimized in order to investigate the mechanism of adsorption process. Several kinetic models including lagergren,s pseudo first order, lagergren,s pseudo second order, Intra particle diffusion, Elvoich model, Natarajan and Khalf model, Bhattarcharya and Venkobechar model were used. In addition equilibrium data were fitted on Langmuir, Freundlich, Temkin, Dubihin-Radhushkevich.The kinetic study on Rhodamine-B dye suggest that the adsorption follows pseudo second order kinetics. Adsorption follows the Freundlich isotherm model than other models. The thermodynamic study on Rhodamine-B reveals that the reaction is spontaneous and endothermic. The study reveals that the biomass pine apple peel proved to be an effective alternative and environmentally benign adsorbent for Rhodamine-B removal from aqueous solution.
\end{abstract}

Keywords: Rhodamine-B; Pine apple peel; Adsorption; Kinetics; Isotherm; Thermodynamics

\section{Introduction}

Industrial wastewater is considered as one of the major pollutants of the environment [1]. Colored wastewater is produced by various industries, such as textile, dyeing, pharmaceutical, food, cosmetics and healthcare, paper and leather industries [2, 3]. Many dyes and their breakdown products may be toxic for living organisms. Therefore decolorization of dyes is important before the discharge of effluent. Removal of dye has been attempted extensively using physico-chemical methods such as coagulation, ultra-filtration, electro-chemical adsorption, photo oxidation, activated carbon adsorption, etc [5]. But these technologies are not efficient, satisfactory and also cost effective [4]. Adsorption has been shown to be one of the most promising and extensively used methods for the removal of both inorganic and organic pollutants from contaminated water [6, 7]. Use of low cost, easily available biomaterials for the adsorption of dyes is practiced as an alternative method and several botanical, low cost materials have directly been used as an adsorbent for removal of dyes from wastewater [8,9]. Now days, agricultural waste materials are receiving much more attention as adsorbents for the removal of dyes from waste water due to its low cost and good availability. In the present study, pine apple peel (PAP) has been used as an adsorbent whose results showed good absorption in Rhodamine-B dye aqueous solution [10].

\section{Material And Methods}

\section{Preparation of an adsorbent}

Pineapple biomass was obtained from fruit market in Thanjavur. The pineapples were peeled of using peeler. The peeled pineapple peels (PAP) were washed using tap water followed by double distilled water. After washing the peel pieces were dried under sun light for 72 hours to remove moisture content present. The dried PAP pieces were washing repeatedly with hot water $(700 \mathrm{C})$ to remove any soluble matter present and dried in oven at $850 \mathrm{C}$ for 48 hours. The dried PAP were powdered and sieved through 100 mesh sieves and stored air tight polythene bottles for adsorption experiments.

\section{Chemical Structure Of Rhodamine -B}<smiles>CCN(CC)c1ccc2c(c1)Oc1cc(N(CC)CC)ccc1-c1cc(S(=O)(=O)[O-])ccc1-2</smiles>

Batch Biosorption Studies 
The experiment was carried out by the batch adsorption method in the Erlenmeyer flasks for a predetermined period using orbital shaker. In the adsorption, parameters such as $\mathrm{PH}$, Initial dye concentration, equilibrium time fixation were studied for optimization. The kinetic studies and isotherm study were carried out at different dye concentration 200ppm, 250ppm, $300 \mathrm{ppm}, 350 \mathrm{ppm}, 400 \mathrm{ppm}$ and $450 \mathrm{ppm}$. By keeping temperature constant, at $150 \mathrm{rpm}$ for 2 and half an hours. The mechanism of adsorption was investigated by Lagergren,s pseudo first order, pseudo-second order, Natarajan and Khalf first order, Bhattacharya and Venkobechar first order, Elvoich and Intra particle diffusion models. The isotherm study results were fitted in Langmuir, Freundlich, Temkin, Dubihin-Radushkevich isotherms. The thermodynamics study carried out at three different temperatures 310,320 and $330 \mathrm{~K}$. The measurement of absorbance of colour was done Spectrophotometrically. The equilibrium adsorption capacity was evaluated using the equation

$$
\text { qe }=(\text { Co-Ce) } \mathrm{V} / \mathrm{m}------(1)
$$

Where qe $(\mathrm{mg} / \mathrm{g})$ is the equilibrium adsorption capacity, Co and $\mathrm{Ce}$ is the initial and equilibrium concentrations (mg/L) of Rhodamine- $\mathrm{B}$ dye solution. $\mathrm{V}$ is the volume and $\mathrm{m}$ is the weight of adsorbent.

\section{Results And Discussion}

Effect of pH: Initial pH of dye can influence the adsorption of it on the surface. In the present study pH 1-10 was used to observe the better adsorption with initial concentration of dye $250 \mathrm{ppm}$ with $1 \mathrm{~g} / \mathrm{L}$ PAP as adsorbent dosage. The reaction mixture was agitated for $1 \mathrm{hr}$ at $310 \mathrm{~K}$ with agitating speed ss150rpm.The most favorable adsorption was seen at basic $\mathrm{pH}$ 8, with 107.9 $\mathrm{mg} / \mathrm{g}$ uptake of dye adsorption. It was shown in the Figure 1.

The surface of the adsorbent which may be negatively charged at higher $\mathrm{pH}$,which favored for adsorption of the positively charged dye cations through electrostatic force of attraction. The adsorption of PAP to adsorbent consequently increased with an increase of $\mathrm{pH}$ values $[11,12]$. So, optimum $\mathrm{pH}$ was 8 .

\section{Effect of biosorbent dosage}

At optimum $\mathrm{pH}$ the biosorbent dose were increased from $0.5 \mathrm{~g} / \mathrm{L}$ at intervals of $0.5 \mathrm{~g} / \mathrm{L}$ up to $5.5 \mathrm{~g}$. The reaction mixture was agitated for $1 \mathrm{hr}$ at $310 \mathrm{~K}$ with agitating speed $150 \mathrm{rpm}$. The percentage of removal efficiency was found high at $4 \mathrm{~g} / \mathrm{L}$. So, optimum biosorbent dose was $4 \mathrm{~g} / \mathrm{L}$. It was shown in Figure 2.

Equilibrium time fixation:At optimum $\mathrm{pH} 8$ and biosorbent dose $4 \mathrm{~g} / \mathrm{L}$ the reaction mixture was agitated for 2 and half an hour with regular time intervals of 15 minutes at $310 \mathrm{~K}$. The maximum adsorption was found at 120 minutes. After that there is no increase in adsorption. It was shown in Figure 3.

\section{Kinetic study of adsorption}

The kinetics of Rhodamine-B adsorption by using PAP was studied at different time intervals and different initial concentration of dye. The kinetic parameters are helpful for the prediction of adsorption rate, give important information for designing and modeling the adsorption process [13].

The mechanism of kinetics was investigated by Natarajan and khalf first order, Elvoich model, Bhattacharya and Venkobechar first order, Lagergren's pseudo first order, pseudo second order and intra particle diffusion models. The study was carried at different time intervals up to equilibrium time and at different ppm at $310 \mathrm{~K}$ are shown in Table 1.

The linearized form of Natarajan and Khalf first order kinetics is presented as

$$
\log \left(\mathrm{C}_{0} / \mathrm{C}_{\mathrm{t}}\right)=(\mathrm{K} / 2.303) \mathrm{t}
$$

Where $\mathrm{C}_{0}$ and $\mathrm{C}_{\mathrm{t}}$ are concentration of Rhodamine- $\mathrm{B}$ dye (mg/l) at time zero and time $\mathrm{t}$ respectively. $\mathrm{K}$ is first order adsorption rate constant $\left(\mathrm{min}^{-1}\right)$ which was calculated from slope of the plot $\left(\log \mathrm{C}_{0} / \mathrm{C}_{\mathrm{t}}\right)$ against $\mathrm{t}$ which was given in Table 2 and Figure 4. The $\mathrm{R}^{2}$ value from 0.849 to 0.866 (Table 5) and also does not fit for whole range of contact time so, it does not follow first order kinetics.

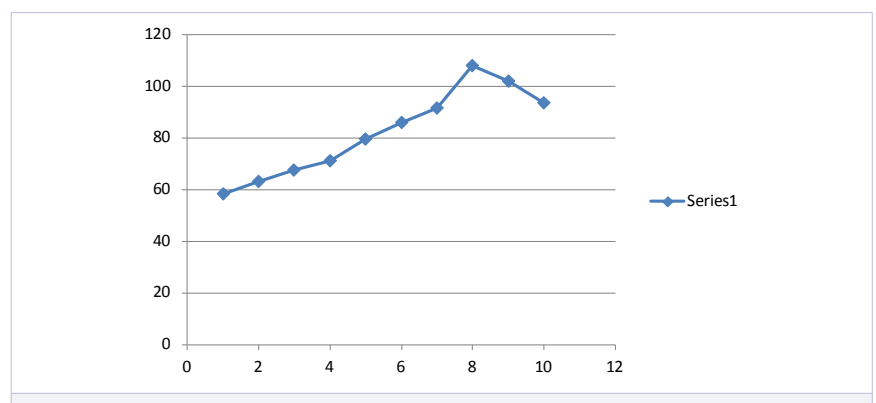

Figure 1

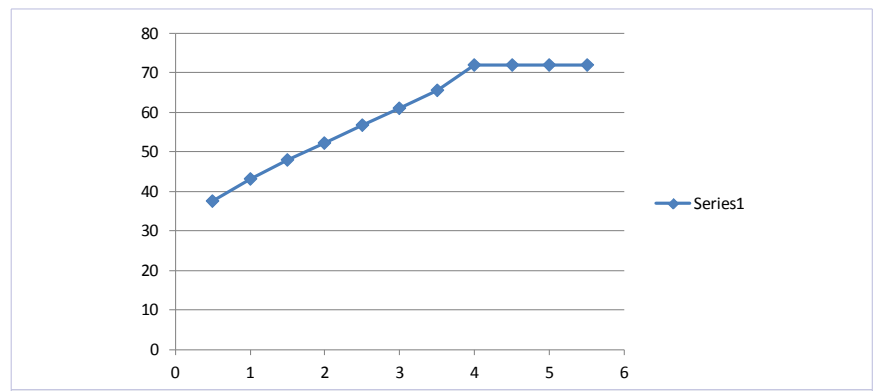

Figure 2

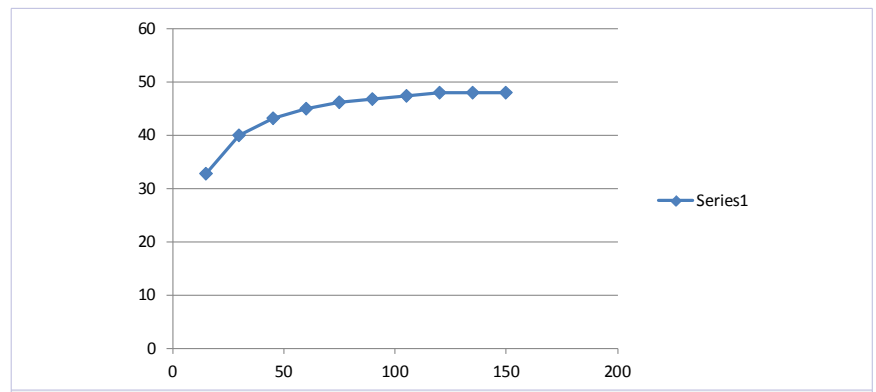

Figure 3 
Table-1

\begin{tabular}{|c|c|c|c|c|c|c|c|c|c|c|c|c|}
\hline \multirow{2}{*}{ Time \ppm } & \multicolumn{2}{|c|}{200} & \multicolumn{2}{|c|}{250} & \multicolumn{2}{|c|}{300} & \multicolumn{2}{|c|}{350} & \multicolumn{2}{|c|}{400} & \multicolumn{2}{|c|}{450} \\
\hline & $C_{t}$ & $q_{t}$ & $C_{t}$ & $q_{t}$ & $C_{t}$ & $q_{t}$ & $C_{t}$ & $q_{t}$ & $C_{t}$ & $q_{t}$ & $C_{t}$ & $q_{t}$ \\
\hline 15 & 94.2 & 26.45 & 118.6 & 32.85 & 142.0 & 39.50 & 168.2 & 45.45 & 194.2 & 51.45 & 220.3 & 57.43 \\
\hline 30 & 70.7 & 32.33 & 89.8 & 40.05 & 108.6 & 47.84 & 129.6 & 55.10 & 150.8 & 62.29 & 172.5 & 69.38 \\
\hline 45 & 60.3 & 34.92 & 77.2 & 43.20 & 94.2 & 51.45 & 112.9 & 59.28 & 132.0 & 67.00 & 151.6 & 74.60 \\
\hline 60 & 54.5 & 36.37 & 70.2 & 44.96 & 86.2 & 53.46 & 103.5 & 61.62 & 122.6 & 69.34 & 139.9 & 77.52 \\
\hline 75 & 50.8 & 37.36 & 65.6 & 46.10 & 81.0 & 54.75 & 97.5 & 63.12 & 114.7 & 71.32 & 132.5 & 79.38 \\
\hline 90 & 48.2 & 37.96 & 62.5 & 46.88 & 77.4 & 55.64 & 93.4 & 64.15 & 110.0 & 72.49 & 127.3 & 80.67 \\
\hline 105 & 46.3 & 38.43 & 60.2 & 47.46 & 74.8 & 56.30 & 90.4 & 64.91 & 106.6 & 73.34 & 123.5 & 81.62 \\
\hline 120 & 44.8 & 38.8 & 58.4 & 47.90 & 72.8 & 56.80 & 88.0 & 65.50 & 104.0 & 74.00 & 120.6 & 82.34 \\
\hline 135 & 44.8 & 38.8 & 58.4 & 47.90 & 72.8 & 56.80 & 88.0 & 65.50 & 104.0 & 74.00 & 120.6 & 82.34 \\
\hline 150 & 44.8 & 38.8 & 58.4 & 47.90 & 72.8 & 56.60 & 88.0 & 65.50 & 104.0 & 74.00 & 120.6 & 82.34 \\
\hline 165 & 44.8 & 38.8 & 58.4 & 47.90 & 72.8 & 56.60 & 88.0 & 65.50 & 104.0 & 74.00 & 120.6 & 82.34 \\
\hline
\end{tabular}

Table-2

\begin{tabular}{|c|c|c|c|c|c|c|}
\hline \multirow{2}{*}{ Time } & 200 ppm & $250 \mathrm{ppm}$ & $300 \mathrm{ppm}$ & $350 \mathrm{ppm}$ & 400 ppm & $450 \mathrm{ppm}$ \\
\hline & $\log C_{0} / C_{t}$ & $\log C_{0} / C_{t}$ & $\log C_{0} / C_{t}$ & $\log C_{0} / C_{t}$ & $\log C_{0} / C_{t}$ & $\log C_{0} / C_{t}$ \\
\hline 15 & 0.327 & 0.3239 & 0.3248 & 0.3183 & 0.3138 & 0.3102 \\
\hline 30 & 0.4517 & 0.4447 & 0.4413 & 0.4315 & 0.4237 & 0.4164 \\
\hline 45 & 0.5205 & 0.5103 & 0.5031 & 0.4914 & 0.4815 & 0.4725 \\
\hline 60 & 0.5644 & 0.5516 & 0.5416 & 0.5291 & 0.5136 & 0.5074 \\
\hline 75 & 0.5955 & 0.581 & 0.5686 & 0.5551 & 0.5425 & 0.531 \\
\hline 90 & 0.6183 & 0.6021 & 0.5884 & 0.5737 & 0.5607 & 0.5484 \\
\hline 105 & 0.6356 & 0.6183 & 0.6032 & 0.5879 & 0.5743 & 0.5615 \\
\hline 120 & 0.6498 & 0.6315 & 0.615 & 0.5996 & 0.585 & 0.5719 \\
\hline
\end{tabular}

Table-3

\begin{tabular}{|c|c|c|c|c|c|c|}
\hline \multirow{2}{*}{ Time } & $\mathbf{2 0 0 p p m}$ & $\mathbf{2 5 0} \mathbf{~ p p m}$ & $\mathbf{3 0 0} \mathbf{~ p p m}$ & $\mathbf{3 5 0} \mathbf{~ p p m}$ & $\mathbf{4 0 0} \mathbf{~ p p m}$ & $\mathbf{4 5 0} \mathbf{~ p p m}$ \\
\cline { 2 - 7 } & $\mathbf{L o g} \mathbf{1}-\mathbf{u}(\mathbf{T})$ & $\mathbf{l o g}-\mathbf{u}(\mathbf{T})$ & $\mathbf{l o g}-\mathbf{u}(\mathbf{T})$ & log-u(T) & log-u(T) & $\mathbf{l o g}-\mathbf{u ( T )}$ \\
\hline 15 & -0.4972 & -0.5028 & -0.5163 & -0.5141 & -0.5161 & -0.519 \\
\hline 30 & -0.7781 & -0.7854 & -0.8024 & -0.7992 & -0.8011 & -0.8024 \\
\hline 45 & -1.0000 & -1.0083 & -1.026 & -1.022 & -1.0241 & -1.0264 \\
\hline 60 & -1.2034 & -1.2104 & -1.2292 & -1.2277 & -1.202 & -1.2321 \\
\hline 75 & -1.4157 & -1.4248 & -1.4425 & -1.4401 & -1.4425 & -1.4425 \\
\hline 90 & -1.6656 & -1.6696 & -1.6947 & -1.6861 & -1.6925 & -1.6925 \\
\hline 105 & -2.0223 & -2.0269 & -2.0969 & -2.0362 & -2.0555 & -2.0555 \\
\hline
\end{tabular}

Table-4

\begin{tabular}{|c|c|c|c|c|c|c|}
\hline logt & $\mathbf{q}_{\mathbf{t}}$ & $\mathbf{q}_{\mathbf{t}}$ & $\mathbf{q}_{\mathbf{t}}$ & $\mathbf{q}_{\mathbf{t}}$ & $\mathbf{q}_{\mathbf{t}}$ & $\mathbf{q}_{\mathbf{t}}$ \\
\hline 1.1761 & 26.45 & 32.85 & 39.5 & 45.45 & 51.45 & 62.29 \\
\hline 1.4471 & 32.33 & 40.05 & 47.84 & 55.1 & 67 & 74.38 \\
\hline 1.6532 & 34.92 & 43.2 & 51.45 & 59.28 & 69.34 & 77.52 \\
\hline 1.7782 & 36.37 & 44.96 & 53.46 & 61.62 & 71.32 & 79.38 \\
\hline 1.8751 & 37.31 & 46.1 & 54.75 & 63.12 & 72.49 & 80.67 \\
\hline 1.9542 & 37.96 & 46.88 & 55.64 & 64.15 & 73.34 & 81.62 \\
\hline 2.0212 & 38.43 & 47.46 & 56.3 & 64.91 & 74 & 82.34 \\
\hline 2.0792 & 38.8 & 47.9 & 56.8 & 65.5 & 7 & \\
\hline
\end{tabular}

\section{Bhattacharya and Venkobechar models}

The linearized form of Bhattacharya and Venkobechar first order kinetic equation is presented as

$\log [1-\mathrm{u}(\mathrm{T})]=-(\mathrm{K} / 2.303) \mathrm{t}$
Where u $(\mathrm{T})=\left[\left(\mathrm{C}_{\mathrm{o}}-\mathrm{C}_{\mathrm{t}}\right) /\left(\mathrm{C}_{\mathrm{o}}-\mathrm{C}_{\mathrm{e}}\right)\right]$

$\mathrm{C}_{\mathrm{e}}$ is equilibrium Rhodamine- $\mathrm{B}$ concentration (mg/l), $\mathrm{K}$ is first order adsorption rate constant $\left(\mathrm{min}^{-1}\right)$ which was calculated from slope of the plot log [1-u(T)] against $t$, which was shown in Table 
3 and Figure 5 . The $\mathrm{R}^{2}$ value is found to be from 0.954 to 0.993 . (Table 5)

\section{ELVOICH MODEL}

The linearized form of Elvoich kinetic equation is presented as [14]

$$
\left.q_{t}=1 / \beta \ln (\alpha, \beta)\right]+\log t / \beta
$$

Where $\alpha$ and $\beta$ are constants calculated from Table 4 and from the intercepts and slopes plot $q_{t}$ against log $t$ shown in Figure 6.The constant $\beta$ is related to the extent of surface coverage. The simple Elvoich models used to describe second order kinetics, assuming of that the actual solid surface is energetically heterogeneous. The Elvoich model has $\mathrm{R}^{2}=0.961$ to 0.964 for adsorbents under study. Where $\alpha$ is initial adsorption rate $1(\mathrm{mg} / \mathrm{g} / \mathrm{min})$ and $\beta$ is related to the extent of the surface coverage and the activation energy for chemisorptions ( $\mathrm{g} \mathrm{mg}^{-1}$ ). The initial adsorption rate, decreased from -0.7885 to -0.7261 while increasing the initial dye concentration from 200 to 450 . It was shown in Figure 6 and table 4 and 5.

\section{Pseudo first order kinetics}

The kinetic data were treated with the following Lagergren's pseudo first order rate equation [15] for $250 \mathrm{ppm}$. Activation energy for chemisorptions $\left(\mathrm{g} \mathrm{mg}^{-1}\right)$.The initial adsorption rate decreased from -0.7855 to -0.7261 , while increasing the initial dye concentration from 200 to $450 \mathrm{ppm}$.

$$
\log \left(\mathrm{q}_{\mathrm{e}}-\mathrm{q}_{\mathrm{t}}\right)=\log \mathrm{qe}-\mathrm{K}_{1} \mathrm{t} /(2.303)-
$$

Where $q_{t}$ and $q_{e}$ are the amount adsorbed at time $t$ and at equilibrium $(\mathrm{mg} / \mathrm{g})$ and $\mathrm{K}_{1}$ is pseudo first order rate constant for the adsorption process $\left(\mathrm{min}^{-1}\right)$. The plot of $\log \left(\mathrm{q}_{\mathrm{e}}-\mathrm{q}_{\mathrm{t}}\right)$ versus $\mathrm{t}$ was shown. (Table 6 Figure 7)

\section{Pseudo second order kinetics}

The pseudo second order model can be represented in the following form [16, 17]

$\mathrm{t} / \mathrm{q}_{\mathrm{t}}=1 / \mathrm{K} 2 \mathrm{q}_{\mathrm{e}} 2+1 \cdot \mathrm{t} / \mathrm{q}_{\mathrm{e}}$

Where $K_{2}$ is the pseudo second order rate constant (g/ mg.min).The plots of $t$ versus $t / q_{t}$ result was shown in Table 7 and Figure 9 for 250ppm. From the above results pseudo first order has $\mathrm{R}^{2}$ value was 0.997 and pseudo second order kinetics has $R^{2}$ value was 1.0000 .for first order $q_{e}$ experimental is 26.06 $\mathrm{mg} / \mathrm{g}$ where as $\mathrm{q}_{\mathrm{e}}$ theoretical is $47.90 \mathrm{mg} / \mathrm{g}$ but for second order $\mathrm{q}_{\mathrm{e}}$ is experimental is 52.63 and $\mathrm{q}_{\mathrm{e}}$ theoretical is $47.90 \mathrm{mg} / \mathrm{g}$.So for the second order only $\mathrm{q}_{e}$ experimental and $\mathrm{q}_{\mathrm{e}}$ theoretical $(\mathrm{mg} / \mathrm{g}$ ) values are nearly same. From this, it clearly indicates that pseudo second order better fitted than pseudo first order.

\begin{tabular}{|c|c|c|c|c|c|c|c|}
\hline \multirow{2}{*}{ Rhodamine B } & \multicolumn{2}{|c|}{ Natarajan and khalf model } & \multicolumn{2}{|c|}{$\begin{array}{c}\text { Bhattacharya and Venkobechar } \\
\text { model }\end{array}$} & \multicolumn{3}{|c|}{ Elvoich model } \\
\hline & $\mathrm{K}\left(\min ^{-1}\right)$ & $\mathbf{R}^{2}$ & $\mathrm{~K}\left(\min ^{-1}\right)$ & $\mathbf{R}^{2}$ & $\alpha(\mathrm{mg} / \mathrm{g} / \mathrm{min})$ & $\beta(\mathrm{g} / \mathrm{mg})$ & $\mathbf{R}^{2}$ \\
\hline 200 & 0.004606 & 0.866 & 0.0368 & 0.992 & -0.7855 & 0.0747 & 0.964 \\
\hline 250 & 0.004606 & 0.861 & 0.0368 & 0.993 & -0.7631 & 0.0613 & 0.963 \\
\hline 300 & 0.004606 & 0.854 & 0.0368 & 0.988 & -0.8035 & 0.0534 & 0.961 \\
\hline 350 & 0.004606 & 0.853 & 0.0368 & 0.993 & -0.7520 & 0.0467 & 0.962 \\
\hline 400 & 0.004606 & 0.855 & 0.0368 & 0.991 & -0.7339 & 0.0409 & 0.964 \\
\hline 450 & 0.004606 & 0.849 & 0.0276 & 0.954 & -0.7261 & 0.0370 & 0.960 \\
\hline
\end{tabular}

Table-5

\section{Table-6}

\begin{tabular}{|c|c|}
\hline Time & Log(qe-qt) \\
\hline 15 & 1.1775 \\
\hline 30 & 0.8949 \\
\hline 45 & 0.6721 \\
\hline 60 & 0.4684 \\
\hline 75 & 0.4684 \\
\hline 90 & 0.0086 \\
\hline 105 & -0.3566 \\
\hline
\end{tabular}

\section{Table-6(a)}

\begin{tabular}{|c|c|}
\hline $\mathrm{K}_{1}$ & -0.015 \\
\hline $\mathrm{q}_{\mathrm{e}}$ (experimental) & 23.77 \\
\hline $\mathrm{R}^{2}$ & 0.997 \\
\hline $\mathrm{q}_{\mathrm{e}}$ (theoretical) & 47.40 \\
\hline
\end{tabular}

\section{Table-7}

\begin{tabular}{|c|c|}
\hline Time & t/qt \\
\hline 15 & 0.4566 \\
\hline 30 & 0.7491 \\
\hline 45 & 1.0417 \\
\hline 60 & 1.3345 \\
\hline 75 & 1.6269 \\
\hline 90 & 1.9198 \\
\hline 105 & 2.2124 \\
\hline 120 & 2.5052 \\
\hline
\end{tabular}

\begin{tabular}{|c|c|}
\hline \multicolumn{1}{|l|}{ Table-7(a) } \\
\hline $\mathrm{K}_{1}$ & 0.0002 \\
\hline $\mathrm{q}_{\mathrm{e}}$ (experimental) & 52.63 \\
\hline $\mathrm{R}^{2}$ & 1 \\
\hline $\mathrm{q}_{\mathrm{e}}$ (theoretical) & 47.90 \\
\hline
\end{tabular}


Table 8:

\begin{tabular}{|c|c|}
\hline${ }^{\mathbf{1}} / 2$ & $\mathbf{q}_{\mathbf{t}}$ \\
\hline 3.873 & 32.85 \\
\hline 5.4772 & 40.05 \\
\hline 6.7082 & 43.2 \\
\hline 7.746 & 44.96 \\
\hline 8.6603 & 46.1 \\
\hline 9.4868 & 46.88 \\
\hline 10.247 & 47.46 \\
\hline 10.9544 & 47.9 \\
\hline
\end{tabular}

\section{Table-8(a)}

\begin{tabular}{|c|c}
\hline K diff & 1.9648 \\
\hline $\mathrm{C}$ & 28.10 \\
\hline $\mathrm{R}^{2}$ & 0.8865 \\
\hline
\end{tabular}

\section{Table-9}

\begin{tabular}{|c|c|}
\hline $\mathbf{C}_{\mathbf{e}}$ & $\mathbf{C e} / \mathbf{q e}$ \\
\hline 44.8 & 1.1217 \\
\hline 58.4 & 1.2192 \\
\hline 72.8 & 1.2817 \\
\hline 88 & 1.3435 \\
\hline 104 & 1.4045 \\
\hline 120.6 & 1.4645 \\
\hline
\end{tabular}

\begin{tabular}{|c|c|}
\hline \multicolumn{2}{|c|}{ Table-9(a) } \\
\hline $\mathrm{q}_{\max }$ & 250 \\
\hline $\mathrm{K}_{\mathrm{L}}$ & 0.00421 \\
\hline $\mathrm{R}_{\mathrm{L}}$ & 0.3458 \\
\hline $\mathrm{R}^{2}$ & 0.984 \\
\hline
\end{tabular}

\begin{tabular}{|c|c|}
\hline \multicolumn{2}{|c|}{ Table-10 } \\
\hline $\log \mathbf{C}_{\mathbf{e}}$ & $\log \mathbf{q}_{\mathbf{e}}$ \\
\hline 1.6513 & 1.5658 \\
\hline 1.7664 & 1.6803 \\
\hline 1.8621 & 1.7543 \\
\hline 1.9444 & 1.8162 \\
\hline 2.017 & 1.8692 \\
\hline 2.0813 & 1.9157 \\
\hline
\end{tabular}

\begin{tabular}{|c|c|}
\hline $1 / \mathrm{n}_{\mathrm{f}}$ & 0.8021 \\
\hline $\mathrm{n}_{\mathrm{f}}$ & 1.2467 \\
\hline $\mathrm{R}^{2}$ & 0.9956 \\
\hline $\mathrm{K}_{\mathrm{f}}$ & 1.7924 \\
\hline
\end{tabular}

\section{Table-11}

\begin{tabular}{|c|c|}
\hline logCe & Qe \\
\hline 1.6513 & 36.8 \\
\hline 1.7664 & 47.9 \\
\hline 1.8621 & 56.8 \\
\hline 1.9444 & 65.5 \\
\hline 2.0170 & 74.0 \\
\hline 2.0813 & 82.35 \\
\hline
\end{tabular}

\begin{tabular}{|c|c|}
\hline Table-11(a) & \multicolumn{2}{|c|}{} \\
\hline $\mathrm{R}^{2}$ & 0.9940 \\
\hline $\mathrm{b}_{\mathrm{t}}$ & 24.24 \\
\hline $\mathrm{A}_{\mathrm{t}}$ & 0.0483 \\
\hline $\mathrm{B}$ & 106.32 \\
\hline
\end{tabular}

\section{Table-12}

\begin{tabular}{|c|c|}
\hline $\boldsymbol{\varepsilon}^{2}$ & $\log \mathbf{q}_{\mathbf{e}}$ \\
\hline 609.6 & 1.5658 \\
\hline 360.24 & 1.6803 \\
\hline 231.95 & 1.7543 \\
\hline 158.50 & 1.8162 \\
\hline 114.28 & 1.8698 \\
\hline 85.56 & 1.9157 \\
\hline
\end{tabular}

\section{Intra particle diffusion}

According to Weber and Morris the intra particle diffusion constant $\left(\mathrm{K}_{\mathrm{i}}\right)$ is given by the following equation [17] for $250 \mathrm{ppm}$.

$$
\mathrm{q}_{\mathrm{t}}=\mathrm{K}_{\mathrm{i}} \mathrm{t} 1 / 2
$$

The intra particle diffusion would be the controlling step if this line passed through the origin .when the plots do not pass through the origin. This is indicative of some degree of boundary layers control and this further show that the intra particle diffusion is not the only rate controlling step but also other processes may control the rate of adsorption [18].

$\mathrm{K}_{\mathrm{i}}\left(\mathrm{mg} / \mathrm{g} / \mathrm{min}^{1 / 2}\right)$ values can be determined from Table 8 and from the Figure 9 , slope of plot $q_{t}$ against $t^{1 / 2}$.The $R^{2}$ value was 0.8865 .

\section{Adsorption isotherm}

Equilibrium isotherm equations are used to describe the experimental adsorption data. The parameters obtained from the different models provide important information on the adsorption mechanism and the surface properties and affinities of the adsorbent. Linear regression is frequently used to determine the best fitting isotherm and the applicability of isotherm equation is compared by judging the correlation co-efficient.

\section{Langmuir isotherm}

The Langmuir equation [18] is expressed as

$$
\mathrm{C}_{\mathrm{e}} / \mathrm{q}_{\mathrm{e}}=\left(1 / \mathrm{K}_{\mathrm{L}} \mathrm{qm}\right)+(1 \mathrm{qm}) \mathrm{C}_{\mathrm{e}}-
$$

Where $\mathrm{q}_{\mathrm{m}}$ is monolayer adsorption capacity $(\mathrm{mg} / \mathrm{g}) \mathrm{K}_{\mathrm{L}}$ is Langmuir isotherm constant related to the affinity of the binding sites and the energy of adsorption $(\mathrm{l} / \mathrm{mg})$. The values are the $\mathrm{q}_{\mathrm{m}}$ and $\mathrm{K}_{\mathrm{L}}$ can be calculated by plotting $\mathrm{C}_{\mathrm{e}} / \mathrm{q}_{\mathrm{e}}$ versus $\mathrm{C}_{\mathrm{e}}$. The dimensionless constant separation factor is $\mathrm{R}_{\mathrm{L}}$ [19]

$$
\mathrm{R}_{\mathrm{L}}=1 /\left(1+\mathrm{K}_{\mathrm{L}} \mathrm{C}_{\mathrm{e}}\right)
$$

The $\mathrm{R}_{\mathrm{L}}$ value indicates the type of isotherm to be either unfavorable $\left(R_{\mathrm{L}}>1\right)$, linear $\left(\mathrm{R}_{\mathrm{L}}=1\right)$, favorable $\left(0<\mathrm{R}_{\mathrm{L}}<1\right)$ or is reversible $\left(R_{L}=0\right)[20,21]$. It was shown in figure 10 and table 9. The $\mathrm{R}^{2}$ value was 0.9847 and $\mathrm{R}_{\mathrm{L}}$ is 0.34548 . So this isotherm is 
Table-13: 310K Temperature.

\begin{tabular}{|c|c|c|c|c|c|c|c|c|c|c|c|c|}
\hline \multirow[b]{2}{*}{ Time $\backslash p p m$} & \multicolumn{2}{|c|}{200} & \multicolumn{2}{|c|}{250} & \multicolumn{2}{|c|}{300} & \multicolumn{2}{|c|}{350} & \multicolumn{2}{|c|}{400} & \multicolumn{2}{|c|}{450} \\
\hline & $C_{t}$ & $\mathbf{q}_{\mathrm{t}}$ & $C_{t}$ & $q_{t}$ & $C_{t}$ & $q_{t}$ & $C_{t}$ & $\mathbf{q}_{\mathrm{t}}$ & $C_{t}$ & $q_{t}$ & $C_{t}$ & $\mathbf{q}_{\mathrm{t}}$ \\
\hline 15 & 94.2 & 26.45 & 118.6 & 32.85 & 142.0 & 39.50 & 168.2 & 45.45 & 194.2 & 51.45 & 220.3 & 57.43 \\
\hline 45 & 60.3 & 34.92 & 77.2 & 43.20 & 94.2 & 51.45 & 112.9 & 59.28 & 132.0 & 67.00 & 151.6 & 74.60 \\
\hline 60 & 54.5 & 36.37 & 70.2 & 44.96 & 86.2 & 53.46 & 103.5 & 61.62 & 122.6 & 69.34 & 139.9 & 77.52 \\
\hline 75 & 50.8 & 37.36 & 65.6 & 46.10 & 81.0 & 54.75 & 97.5 & 63.12 & 114.7 & 71.32 & 132.5 & 79.38 \\
\hline 90 & 48.2 & 37.96 & 62.5 & 46.88 & 77.4 & 55.64 & 93.4 & 64.15 & 110.0 & 72.49 & 127.3 & 80.67 \\
\hline 105 & 46.3 & 38.43 & 60.2 & 47.46 & 74.8 & 56.30 & 90.4 & 64.91 & 106.6 & 73.34 & 123.5 & 81.62 \\
\hline 120 & 44.8 & 38.8 & 58.4 & 47.90 & 72.8 & 56.80 & 88.0 & 65.50 & 104.0 & 74.00 & 120.6 & 82.34 \\
\hline 135 & 44.8 & 38.8 & 58.4 & 47.90 & 72.8 & 56.80 & 88.0 & 65.50 & 104.0 & 74.00 & 120.6 & 82.34 \\
\hline 150 & 44.8 & 38.8 & 58.4 & 47.90 & 72.8 & 56.60 & 88.0 & 65.50 & 104.0 & 74.00 & 120.6 & 82.34 \\
\hline 165 & 44.8 & 38.8 & 58.4 & 47.90 & 72.8 & 56.60 & 88.0 & 65.50 & 104.0 & 74.00 & 120.6 & 82.34 \\
\hline
\end{tabular}

Table-14: $320 \mathrm{~K}$ Temperature.

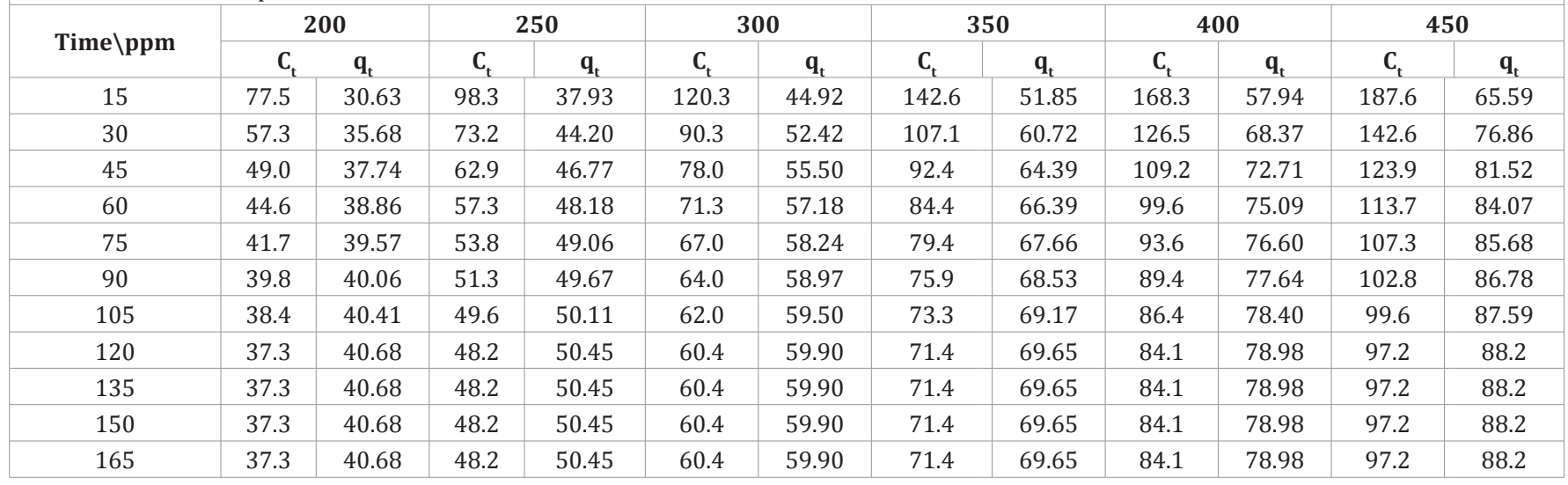

Table 15: 330K Temperature.

\begin{tabular}{|c|c|c|c|c|c|c|c|c|c|c|c|c|}
\hline \multirow{2}{*}{ Time \ppm } & \multicolumn{2}{|c|}{200} & \multicolumn{2}{|c|}{250} & \multicolumn{2}{|c|}{300} & \multicolumn{2}{|c|}{350} & \multicolumn{2}{|c|}{400} & \multicolumn{2}{|c|}{450} \\
\hline & $C_{t}$ & $\mathbf{q}_{\mathrm{t}}$ & $\mathrm{C}_{\mathrm{t}}$ & $q_{t}$ & $\mathrm{C}_{\mathrm{t}}$ & $q_{t}$ & $\mathrm{C}_{\mathrm{t}}$ & $q_{t}$ & $C_{t}$ & $q_{t}$ & $\mathrm{C}_{\mathrm{t}}$ & $q_{t}$ \\
\hline 15 & 60.3 & 34.93 & 78.2 & 42.95 & 98.6 & 50.40 & 119.5 & 57.62 & 141.8 & 64.54 & 163.6 & 71.60 \\
\hline 30 & 44.0 & 39.00 & 58.5 & 47.87 & 72.7 & 56.83 & 88.6 & 65.34 & 105.7 & 73.57 & 122.8 & 81.79 \\
\hline 45 & 37.8 & 40.56 & 51.0 & 49.76 & 62.5 & 59.38 & 76.4 & 68.39 & 91.4 & 77.16 & 106.6 & 85.86 \\
\hline 60 & 34.4 & 41.39 & 48.3 & 50.42 & 57.0 & 60.74 & 69.9 & 70.03 & 83.6 & 79.09 & 97.8 & 88.05 \\
\hline 75 & 32.4 & 41.91 & 44.4 & 51.39 & 53.7 & 61.58 & 65.8 & 71.05 & 78.8 & 80.30 & 92.3 & 89.42 \\
\hline 105 & 30.0 & 42.51 & 41.5 & 52.12 & 49.7 & 62.58 & 61.0 & 72.25 & 73.1 & 81.72 & 85.8 & 91.04 \\
\hline 120 & 29.3 & 42.68 & 38.2 & 52.95 & 48.4 & 62.89 & 59.4 & 72.64 & 71.3 & 82.18 & 83.8 & 91.56 \\
\hline 135 & 29.3 & 42.68 & 38.2 & 52.95 & 48.4 & 62.89 & 59.4 & 72.64 & 71.3 & 82.18 & 83.8 & 91.56 \\
\hline 150 & 29.3 & 42.68 & 38.2 & 52.95 & 48.4 & 62.89 & 59.4 & 72.64 & 71.3 & 82.18 & 83.8 & 91.56 \\
\hline 165 & 29.3 & 42.68 & 38.2 & 52.95 & 48.4 & 62.89 & 59.4 & 72.64 & 71.3 & 82.18 & 83.8 & 91.56 \\
\hline
\end{tabular}

favorable.

\section{Freundlich Isotherm}

The isotherm was represented by

$\log \mathrm{q}_{\mathrm{e}}=\log \mathrm{K}_{\mathrm{f}}+1 / \mathrm{n} \log \mathrm{C}_{\mathrm{e}}$

Where $\mathrm{q}_{\mathrm{e}}$ is the amount of Rhodamine - B adsorbed at the equilibrium (mg/g), $\mathrm{C}_{\mathrm{e}}$ is the equilibrium constant of Rhodamine - $\mathrm{B}$ in solution $(\mathrm{mg} / \mathrm{L})[22,23] . \mathrm{K}_{\mathrm{f}}$ and $1 / \mathrm{n}_{\mathrm{f}}$ are constant incorporating factor affecting the adsorption capacity and intensity of adsorption respectively.It was shown in the Figure 11 and table 10 . The $\mathrm{R}^{2}$ value is 0.9956 .It indicates good linearity and obeys the Freundlich isotherm.

\section{Temkin isotherm}

The Temkin isotherm is given as $q_{e}=\beta \ln A+\beta \ln C_{e}$ 


\begin{tabular}{|c|c|c|c|c|c|c|c|c|}
\hline \multicolumn{9}{|c|}{ Table-16 } \\
\hline \multirow{2}{*}{ ppm } & \multicolumn{2}{|c|}{$310 \mathrm{~K}$} & \multicolumn{2}{|c|}{$320 \mathrm{~K}$} & \multicolumn{2}{|c|}{$330 \mathrm{~K}$} & \multirow{2}{*}{$\begin{array}{c}\Delta \mathrm{H} \\
\mathrm{KJ} / \mathrm{mol}\end{array}$} & \multirow{2}{*}{$\begin{array}{c}\Delta \mathrm{S} \\
\mathrm{J} / \mathrm{mol}\end{array}$} \\
\hline & $\mathrm{K}_{0}$ & $\Delta \mathrm{G}^{0}$ & $\mathrm{~K}_{0}$ & $\Delta \mathrm{G}^{0}$ & $\mathbf{K}_{0}$ & $\Delta \mathrm{G}^{0}$ & & \\
\hline 200 & 3.4643 & -3201.40 & -4.3619 & -4.3619 & 5.8259 & -4835.11 & 21608.09 & 80.04 \\
\hline 250 & 3.2808 & -3062.10 & -4.1867 & -4.1867 & 5.5445 & -4699.29 & 21807.62 & 80.27 \\
\hline 300 & 3.1203 & -2933.32 & -3.9669 & -3.9669 & 5.1984 & -4522.44 & 21209.01 & 77.92 \\
\hline 350 & 2.9773 & -2811.92 & -3.9019 & -3.9019 & 4.8923 & -4355.94 & 19887.09 & 73.50 \\
\hline 400 & 2.8462 & -2695.85 & -3.7562 & -3.7562 & 4.6101 & -4193.9 & 20045.05 & 73.55 \\
\hline 450 & 2.7313 & -2589.65 & -3.6296 & -3.6296 & 4.3699 & -4046.12 & 19537.90 & 71.59 \\
\hline
\end{tabular}

\begin{tabular}{|c|c|c|c|}
\hline \multicolumn{4}{|c|}{ Table-17 } \\
\hline \multirow{2}{*}{$\mathbf{p p m}$} & $\mathbf{3 1 0} \mathbf{K}$ & $\mathbf{3 2 0} \mathbf{K}$ & $\mathbf{3 3 0} \mathbf{~}$ \\
\cline { 2 - 4 } & $\mathbf{l n} \mathbf{K}_{\mathbf{0}}$ & $\mathbf{l n} \mathbf{K}_{\mathbf{0}}$ & $\mathbf{l n} \mathbf{K}_{\mathbf{0}}$ \\
\hline 200 & 1.2425 & 1.4729 & 1.7623 \\
\hline 250 & 1.1881 & 1.4319 & 1.7128 \\
\hline 300 & 1.1381 & 1.3779 & 1.6483 \\
\hline 350 & 1.1091 & 1.3614 & 1.5876 \\
\hline 400 & 1.0459 & 1.3234 & 1.5282 \\
\hline 450 & 1.0047 & 1.2891 & 1.4747 \\
\hline
\end{tabular}

Where $A_{t}(l / g)$ is the equilibrium binding constant corresponding to the maximum binding energy and constant $\mathrm{B}$ is related to heat of adsorption [25]. A Linear plots of $q_{e}$ against $l n$ $\mathrm{C}_{\mathrm{e}}$. Enables the determination of constant $\mathrm{B}$ and $\mathrm{A}$ from slope and intercept (table 11 and Fig 12).The $\mathrm{R}^{2}$ value is 0.994 .

\section{Dubihin - Radushkevich isotherm}

The Dubihin- Radushkevich isotherm is given as $--(11)$

$\ln Q=\ln Q m-K^{\prime}\left[R T \ln \left(1+\left(1 / c_{e}\right)\right] 2 E=-(2 k)^{-0.5}\right.$

This adsorption curve depends of the adsorbent pores. (26) The plot of $\operatorname{lnq}_{\mathrm{e}}$ vs. $\varepsilon^{2}$ for Rhodamine - B are shown in table 13 and figure13. The mean adsorption energy (E) gives information about the chemical and physical nature of adsorption. The $\mathrm{R}^{2}$ value is 0.955 .

\section{Thermodynamic Analysis}

Thermodynamic parameters such as change in free energy $\Delta \mathrm{G}$ $(\mathrm{J} /$ mole), enthalpy $\Delta \mathrm{H},(\mathrm{J} /$ mole) $\Delta \mathrm{S}(\mathrm{J} / \mathrm{K} / \mathrm{mole})$ were determined using following equations

$$
\begin{aligned}
& \mathrm{K}_{0}=\mathrm{C}_{\text {solid }} / \mathrm{C}_{\text {liquid }} \text {--------- (12) } \\
& \Delta \mathrm{G}=-\mathrm{RT} \ln \mathrm{K}_{0}- \\
& \Delta \mathrm{G}=\Delta \mathrm{H}-\mathrm{T} \Delta \mathrm{S} \\
& \text { In } \mathrm{k}_{0}=-\Delta \mathrm{G} / \mathrm{RT} \\
& \ln \mathrm{k}_{0}=-\Delta \mathrm{S} / \mathrm{R}-\Delta \mathrm{H} / \mathrm{R}
\end{aligned}
$$

Where $\mathrm{K}_{\mathrm{o}}$ is equilibrium constant, $\mathrm{C}_{\text {solid }}$ is solid phase concentration at equilibrium $(\mathrm{mg} / \mathrm{L}), \mathrm{C}_{\text {liquid }}$ is liquid phase concentration at equilibrium $(\mathrm{mg} / \mathrm{L}), \mathrm{T}$ is absolute temperature in Kelvin and $\mathrm{R}$ is a gas constant. $\Delta \mathrm{G}$ values obtained from equation (13), It was presented in Table $(14,15,16,17)$.

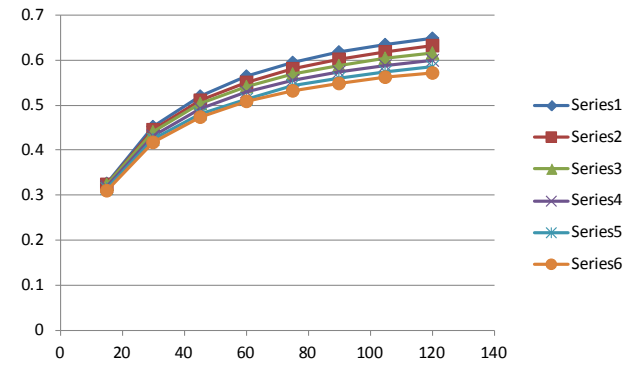

Figure 4

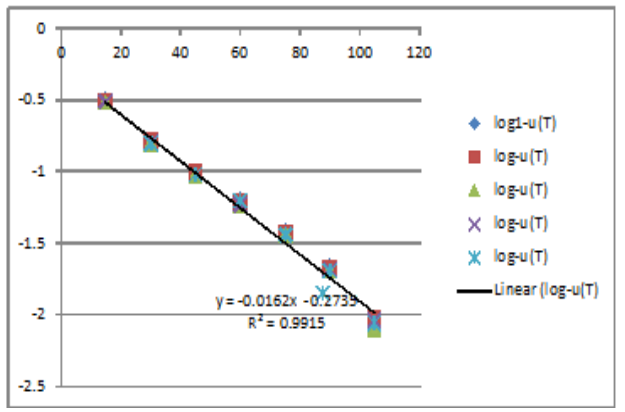

Figure 5
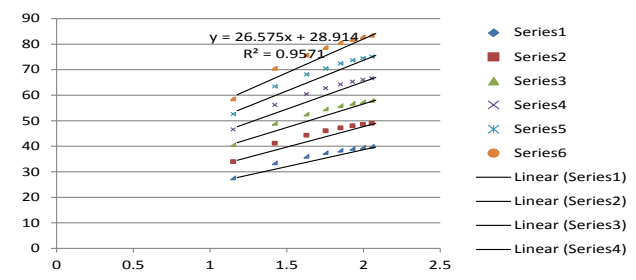

Figure 6

\section{Conclusion}

The aim of this paper was utilization of natural biosorbent PAP as adsorbent for the removal of Rhodamine-B dye. Even though Bhattarchaya and Venkobechar frist order kinectic model,lagergren's pseudo first order kinetic model gave better results ,but pseudo second order kinetic model was best fitted kinetic of adsorption. The correlation co efficient $\mathrm{R}^{2}=1$ for second order adsorption model and $\mathrm{q}_{\mathrm{e}}$ theoretical values are consistent with $q_{e}$ experimental value showed that pseudo 


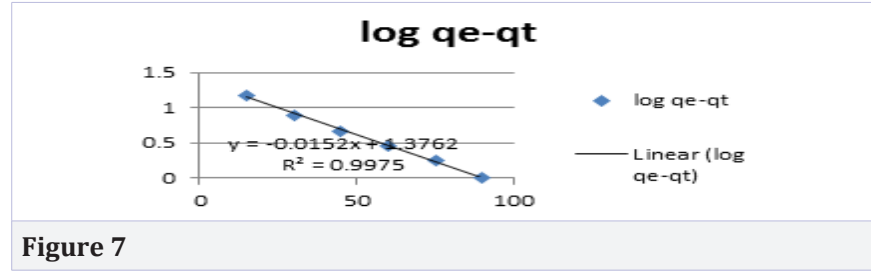

Figure 7

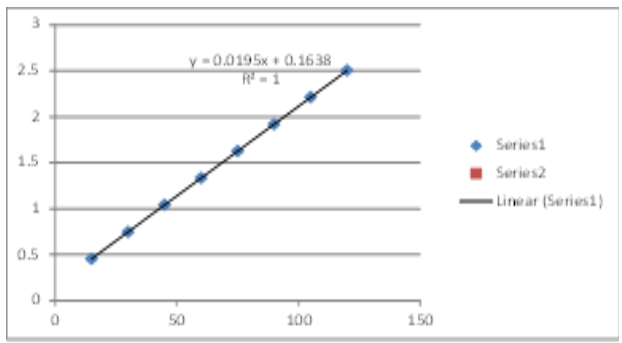

Figure 8

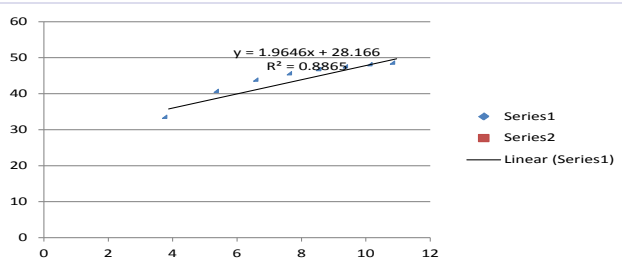

Figure 9

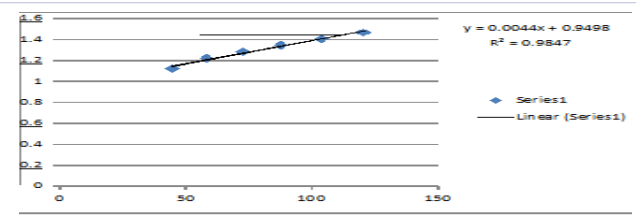

Figure 10

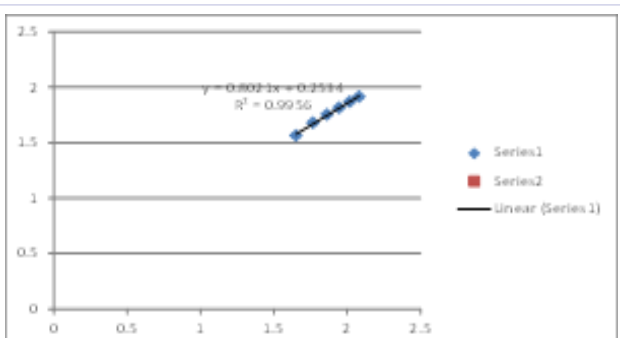

Figure 11

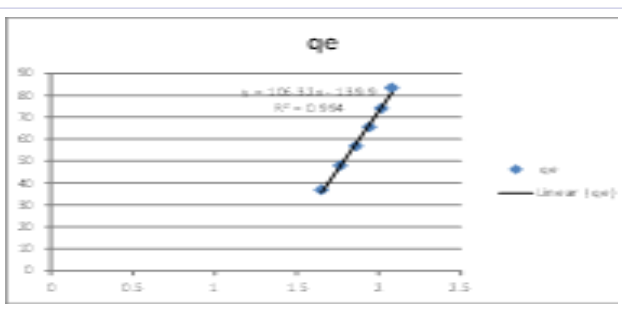

Figure 12

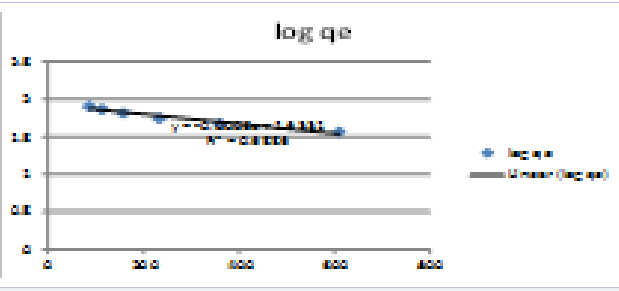

Figure 13

second order adsorption equation fit with whole range of contact time. Among isotherms, Freundlich isotherm was found to be best fitting model with respect to $\mathrm{R}^{2}$ values. $\Delta \mathrm{G}, \Delta \mathrm{H}$ and $\Delta \mathrm{S}$ values showed that favorable, spontaneous and endothermic. The PAP adsorbents have excellent adsorption capacity compare to any other non conventional adsorption. So PAP can be used as a low cost attractive alternative for costly activated carbon.

\section{References}

1. Sapci Z Ustyb b. The removal of color and COD from textile wastewater by using waste pumice. Elec j Environ Agric Food Che. 2003;2(2):286290.

2. Samarghandi MR, Zarrabi M, Sepehr MN, Amrane a. Application of acidic treated pumice as an adsorbent for the removal of azo dye from aqueous solutions; kinetic, equilibrium and thermodynamic studies. Iranian j Environ Health Sci Eng. 2012;9(1):9.

3. Zahra Derakhshan, Mohammad Ali baghapour, Modeh ranjbar, Mohammad Raramarzian. Adsorption of Methylene blue dye from aqueous solutions by modified pumice stone: kinetics and equilibrium Studies. Health Scope. 2013;2(3):136-144. DOI: 10.17795/ jhealthscope- 12492

4. Iqbal MJ, Ashiq MN. Adsorption of dyes from aqueous solutions on activated charcoal. 2007;139(1):57-66.

5. Kannan $\mathrm{N}$ and sundaram M. Kinetics and Mechanism of removal of Methylene blue by adsorption on Carbons: a comparative Study. Dyes and Pigments.2001;5(1):25-40.

6. KG Bhattacharyya, A Sharma. Azadirach Indica, -Leaf Powder as an effective biosorbent for dyes . J Environ Management. 2004;71:217229.

7. Jayaraj R, Chandramohan M, Martin Deva Prasath and Khant H. Malachite green dye removal using seaweed Interomorpha. J Che. 2011;8(2):649-656.

8. Ashly leena prasad and Thirumalaisamy. Adsorption of hazardous cationic dyes from aqueous solution on to acacia nilotica leaves as an eco friendly adsorbent, Sustain environ res. 2012;22(2):113-122.

9. Zhang j, Yan Li and Zhang c. Adsorption of Malachite green from aqueous solution onto carbon prepared from Arundo donax root. J Hazard Mater. 2008;150(3):774-782.

10. Al-ghouti ma, khraisheh mam, allen sj, ahmad mn. The Removal of dyes from textile wastewater; A study of The physical characteristics and adsorption mechanisms of diatomaceous Earth. J Environ Manag. 2003;69(3):229-238.

11. Ashly leena prasad and Thirumalaisamy. Adsorption of hazardous cationic dyes from aqueous solution on to acacia nilotica leaves as an eco friendly adsorbent, Sustain environ res. 2012;22(2):113-122.

12. Pan X, Zhang D. Removal of Malachite green from water by firmiana 
simplex wood fiber. Electron J Biotechnol. 2009;12(4):1.

13. Back MH, Ijagbemi CO, Jin OS, Kim DS. Removal of malachite green from aqueous solution using degreased coffee bean. J Hazard Mater 2010;176(1-3):820-828. doi: 10.1016/j.jhazmat.2009.11.110

14. Rashid Mahmaood and Irtaza javania. Adsorption of commercial dye (red-cis-bar) onto ash collected from brick kiln. World Appl Sci Journal. 2014;29(8):968-977.

15. Ho YS, Mc kay G. The sorption of Lead (ii) Ions on Peat. Water Res 1999;33(2):578-584

16. Ho YS, Mc kay G. Sorption of dye from aqueous solution by peat. Che Eng j. 1998;70(2):115-124. doi:10.1016/S0923-0467(98)00076-1

17.Zhang J, LI Y, Zhang C, Jing y. Adsorption of malachite green from aqueous solution onto carbon prepared from arundo donax root. J Hazard Mater.2008;150(3):774-782.

18. Satish patil, Vaijantha Deshmukh, Sameer Renukdas, Naseema patel Kinetics of adsorption of crystal violet from aqueous solution using difference natural materials. Inter J Environ Sci. 2011 ;1(6):1116.

19. Saha P, Chowdhury S, Gupta S, Kumar J, Kumar R. Assessment on the removal of malachite green using tamarind fruit shell as biosorbent Clean Soil Air Water. 2010;38(5-6):437-445.

20. Hall KR, Eagleton IC, Acrivos A, Vermealen t. Pore solid diffusion kinetics in fixed bed adsorption under constant pattern conditions. Ind-eng che fundam.1966;5(2):212-223. DOI: 10.1021/i160018a011

21. N Dhutson and RT Yang. Adsorption. J Colloid Interf Sci. 2000;188.

22. MI Tempkin, V Pyzhev. Kinetics of ammonia synthesis on promotediron catalyst. Acta Phys Chim Vssr. 1940;12:327-356.

23. C Aharoni, M Ungarish. Kinetics of activated chemisorptions theoritical models. J che soc faraday trans. 1997;73: 456-464.

24. A. Gunany e Arslankaya, I Tosun clino. J hazard mater. 2007;146:362371.

25. A Dabrowski. Adsorption from theory to Practice. Adv Colloid Interface sci. 2001;93(1-3):135-224.

26. Rajashree kobiraj, Neha gupta, Atul kumar kushwata and MC Chattopadhyaya. Determination of equilibrium, kinetic and thermodynamic parameters for the adsorption of brilliant green dye from aqueous solutions onto eggshell powder. Ind J Chem Tech. 2012;19(1):26-31. 